\title{
Timing of in-hospital cardiac arrest after pediatric cardiac surgery: An important metric for quality improvement and prognostication?
}

\author{
Misha Dagan, MD, ${ }^{\mathrm{a}, \mathrm{b}, \mathrm{c}}$ Warwick Butt, MBBS, ${ }^{\mathrm{a}, \mathrm{b}, \mathrm{c}}$ Yves d'Udekem, MD, PhD, ${ }^{\mathrm{b}, \mathrm{c}, \mathrm{d}}$ and \\ Siva P. Namachivayam, MBBS, MEpi, ${ }^{a, b, c}$ Melbourne, Australia
}

\footnotetext{
From the ${ }^{\mathrm{a}}$ Intensive Care Unit and ${ }^{\mathrm{d}}$ Cardiac Surgery, Royal Children's Hospital, Melbourne, Australia; ${ }^{\mathrm{b}}$ Murdoch Children's Research Institute, Melbourne, Australia; and ${ }^{\mathrm{c}}$ Department of Paediatrics, University of Melbourne, Australia.

Mr Namachivayam is supported by a health professional research scholarship (award No: 101003) from the National Heart Foundation of Australia. Dr d'Udekem receives personal fees from MSD, Actelion.

Received for publication Sept 15, 2018; revisions received Dec 12, 2018; accepted for publication Jan 18, 2019; available ahead of print Feb 20, 2019.

Address for reprints: Siva P. Namachivayam, MBBS, MEpi, Intensive Care Unit, The Royal Children's Hospital, Flemington Rd, Parkville 3052, Australia (E-mail: siva.namachivayam@rch.org.au).

J Thorac Cardiovasc Surg 2019;157:e401-6 0022-5223/\$36.00

Crown Copyright $\odot 2019$ Published by Elsevier Inc. on behalf of The American Association for Thoracic Surgery https://doi.org/10.1016/j.jtcvs.2019.01.066
}

Supplemental material is available online.

In-hospital-cardiac-arrest (IHCA) occurs in 3\% to $4 \%$ of children recovering from cardiac surgery with an associated mortality of $35 \%$ to $40 \%$; a high proportion of survivors experience long-term neurodevelopmental disability. ${ }^{1,2}$ Understanding patterns of IHCA timing and its association with outcome can potentially lead to the development of risk stratification tools and targeted management protocols. Currently there are no reports linking timing of IHCA after pediatric cardiac surgery and outcome. We therefore studied the association between timing of IHCA and hospital survival in a cohort of children undergoing cardiac surgery.

\section{MATERIALS AND METHODS}

This is a retrospective cohort study conducted at Royal Children's Hospital, Melbourne, Australia (ethics \#DA004-2016-18). Children younger than age 18 years who sustained an IHCA following a risk-adjusted congenital heart surgery procedure between January 2007 and December 2016 were included. Exclusions included medical cardiac patients and IHCA occurring in the operating theatre. Study variables were collected from our Paediatric Intensive Care Unit database and medical records. Cardiac arrest was defined as cessation of cardiac mechanical activity with nonpalpable central pulse requiring cardiac massage for $\geq 1$ minute, as recommended by the Utstein Guidelines. ${ }^{3}$ Primary study outcome was survival to hospital discharge.

Time-to-arrest after surgery was categorized ( $<12$ hours, $12-48$ hours, or $>48$ hours) due to its nonlinear relationship with mortality; categories chosen reflected low cardiac output syndrome secondary to postbypass inflammatory response being prominent within the first 12 hours and arrest events occurring after 48 hours more likely to represent newer complications.

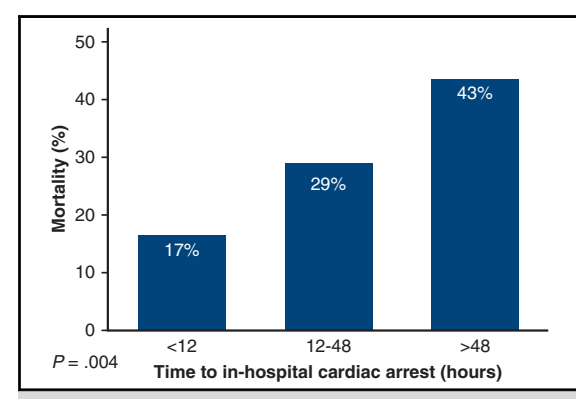

Cardiac arrest mortality by time to arrest in children following cardiac surgery.

Central Message
Cardiac arrest occurring $>48$ hours after car-
diac surgery is associated with higher mortality.
Early identification and prevention of late ar-
rests is imperative to improving overall
survival.

See Commentary page e407.
Categorical variables were analyzed using the $\chi^{2}$ test and continuous variables were analyzed using the Kruskal-Wallis test to compare study characteristics between study groups. A multivariable logistic regression analyses (controlled for age, cardiopulmonary bypass duration, vasoactive inotrope score [VIS], risk-adjusted congenital heart surgery category, and place of arrest) was used to study the association between time-to-arrest and hospital survival. VIS has been shown to have strong associations with cardiac arrest and mortality ${ }^{4}$ and was therefore used in the model. The primary outcome was also analyzed with the Kaplan-Meier productlimit method. Analyses were performed using Stata version 15 (StataCorp, College Station, Tex).

\section{RESULTS}

Incidence of IHCA following cardiac surgery was $4.2 \%$ (160 of 3781 patients). Thirty-eight percent $(n=60)$ of IHCA occurred within 12 hours of cardiac surgery, 19\% $(\mathrm{n}=31)$ at 12 to 48 hours, and $43 \%(\mathrm{n}=69)$ at $>48$ hours. Overall hospital survival following IHCA postcardiac surgery was $69 \%$ (111 out of 160 patients) and 1-year survival was 66\% (105 out of 160 patients). Table 1 details patient characteristics and etiologies of IHCA by time to arrest. Children who survived to hospital discharge were older, with a median age of 28 days (interquartile range [IQR], 6-119 days) versus 7 days (IQR, 3-28 days) $(P<.01)$, had shorter cardiopulmonary 
TABLE 1. Comparison of patient characteristics and etiologies between time to arrest categories in children who experienced in-hospital cardiac arrest (IHCA) following cardiac surgery

\begin{tabular}{|c|c|c|c|c|}
\hline \multirow[b]{2}{*}{ Characteristic } & \multicolumn{3}{|c|}{ Time to IHCA (h) } & \multirow[b]{2}{*}{$P$ value } \\
\hline & $\overline{<12(n=60)}$ & $12-48(n=31)$ & $>48(n=69)$ & \\
\hline Age (d) & $22(6-111)$ & $24(5-83)$ & $14(3-101)$ & .4 \\
\hline Weight (kg) & $3(3-5)$ & $4(3-4)$ & $4(3-5)$ & .5 \\
\hline Male sex & $26(43)$ & $17(55)$ & $31(45)$ & .6 \\
\hline Prematurity & $14(23)$ & $8(26)$ & $13(19)$ & .7 \\
\hline Chromosome/genetic syndrome & $14(23)$ & $9(29)$ & $16(23)$ & .8 \\
\hline $\begin{array}{l}\text { RACHS-1 category } \\
1-2 \\
3-4 \\
5-6\end{array}$ & $\begin{array}{r}9(15) \\
43(72) \\
8(13)\end{array}$ & $\begin{array}{r}8(26) \\
19(61) \\
4(13)\end{array}$ & $\begin{array}{c}5(7) \\
43(62) \\
21(30)\end{array}$ & .02 \\
\hline CPB time (min) & $152(90-196)$ & $142(77-212)$ & $158(100-225)$ & .7 \\
\hline Aortic crossclamp time (min) & $72(28-110)$ & $62(0-118)$ & $70(37-117)$ & .8 \\
\hline Time to arrest (h) & $3.4(1.1-6.6)$ & $24.5(16.8-32.3)$ & $144.2(93.5-447.9)$ & $<.001$ \\
\hline $\begin{array}{l}\text { Location of arrest } \\
\text { PICU } \\
\text { Ward }\end{array}$ & $\begin{array}{c}60(100) \\
0(0)\end{array}$ & $\begin{array}{c}31(100) \\
0(0)\end{array}$ & $\begin{array}{l}53(77) \\
16(23)\end{array}$ & $<.01$ \\
\hline Weekend arrests* & $3(5)$ & $10(32)$ & $19(28)$ & $<.01$ \\
\hline Night time arrests $\dagger$ & $20(33)$ & $8(26)$ & $17(25)$ & .5 \\
\hline Requirement for ECPR & $21(35)$ & $15(48)$ & $34(49)$ & .2 \\
\hline CPR duration $(\min ) \ddagger$ & $9(3-24)$ & $20(3-37)$ & $15(5-35)$ & .1 \\
\hline Admission VIS $\ddagger, \S$ & $5(2-10)$ & $5(2-10)$ & $5(4-10)$ & .4 \\
\hline Admission lactate $(\mathrm{mmol} / \mathrm{L}) \ddagger$ & $2(2-4)$ & $2(2-3)$ & $3(2-4)$ & .6 \\
\hline Intensive care unit LOS (d) & $9(5-14)$ & $16(9-30)$ & $17(11-32)$ & .01 \\
\hline Hospital LOS (d) & $28(14-57)$ & $33(15-77)$ & $40(20-73)$ & .04 \\
\hline Hospital survival & $50(83)$ & $22(71)$ & $39(57)$ & $<.01$ \\
\hline 1 -y survival & $46(77)$ & $22(71)$ & $37(54)$ & .02 \\
\hline Etiology of IHCA & & & & Total \\
\hline LCOS & $12(20)$ & $9(29)$ & $2(3)$ & $23(14)$ \\
\hline Tamponade & $4(7)$ & $5(16)$ & $11(16)$ & $20(13)$ \\
\hline Residual lesion $\|$ & $6(10)$ & $4(13)$ & $10(15)$ & $20(13)$ \\
\hline Surgical complication $\llbracket$ & $13(22)$ & $0(0)$ & 7 (10) & $20(13)$ \\
\hline Arrhythmia/coronary ischemia & $7(12)$ & $4(13)$ & $7(10)$ & $18(11)$ \\
\hline Sepsis & $3(5)$ & $1(3)$ & 13 (19) & $17(11)$ \\
\hline Respiratory arrest & $4(5)$ & $5(16)$ & $7(10)$ & $16(9)$ \\
\hline Pulmonary hypertension & $8(10)$ & $1(3)$ & $7(10)$ & $16(9)$ \\
\hline Other\# & $6(10)$ & $2(7)$ & $5(7)$ & $13(8)$ \\
\hline
\end{tabular}

Values for continuous variables are presented as median (interquartile range), values for categorical variables are presented as n (\%). IHCA, In-hospital cardiac arrest; $R A C H S-1$, risk-adjusted congenital heart surgery category; $C P B$, cardiopulmonary bypass time; $P I C U$, pediatric intensive care unit; $E C P R$, extracorporeal cardiopulmonary resuscitation; $C P R$, cardiopulmonary resuscitation; VIS, vasoactive inotropic score; $L O S$, length of stay; $L C O S$, low cardiac output syndrome. *Weekend defined as 11 pm Friday night to 7 am Monday morning. $\dagger$ Night time arrest defined as between $11 \mathrm{pm}$ and $7 \mathrm{am}$. $\ddagger$ Data available on CPR duration for 158 patients, admission VIS for 151 patients, and admission lactate for 148 patients. $§$ Dopamine dose $(\mu \mathrm{g} / \mathrm{kg} / \mathrm{min})+$ dobutamine dose $(\mu \mathrm{g} / \mathrm{kg} / \mathrm{min})+100 \times$ adrenalin dose $(\mu \mathrm{g} / \mathrm{kg} / \mathrm{min})+10 \times$ milrinone dose $(\mu \mathrm{g} / \mathrm{kg} / \mathrm{min})+10,000 \times$ vasopressin dose $(\mathrm{U} / \mathrm{kg} / \mathrm{min})+100 \times$ noradrenalin dose $(\mu \mathrm{g} / \mathrm{kg} / \mathrm{min})$. $\|$ Requiring unplanned repeat surgical intervention for incomplete repair within the same hospital admission. $\lceil$ Major bleeding or shunt occlusion. \#Unknown, medication related, extracorporeal life support failure, or pacemaker failure to capture. 


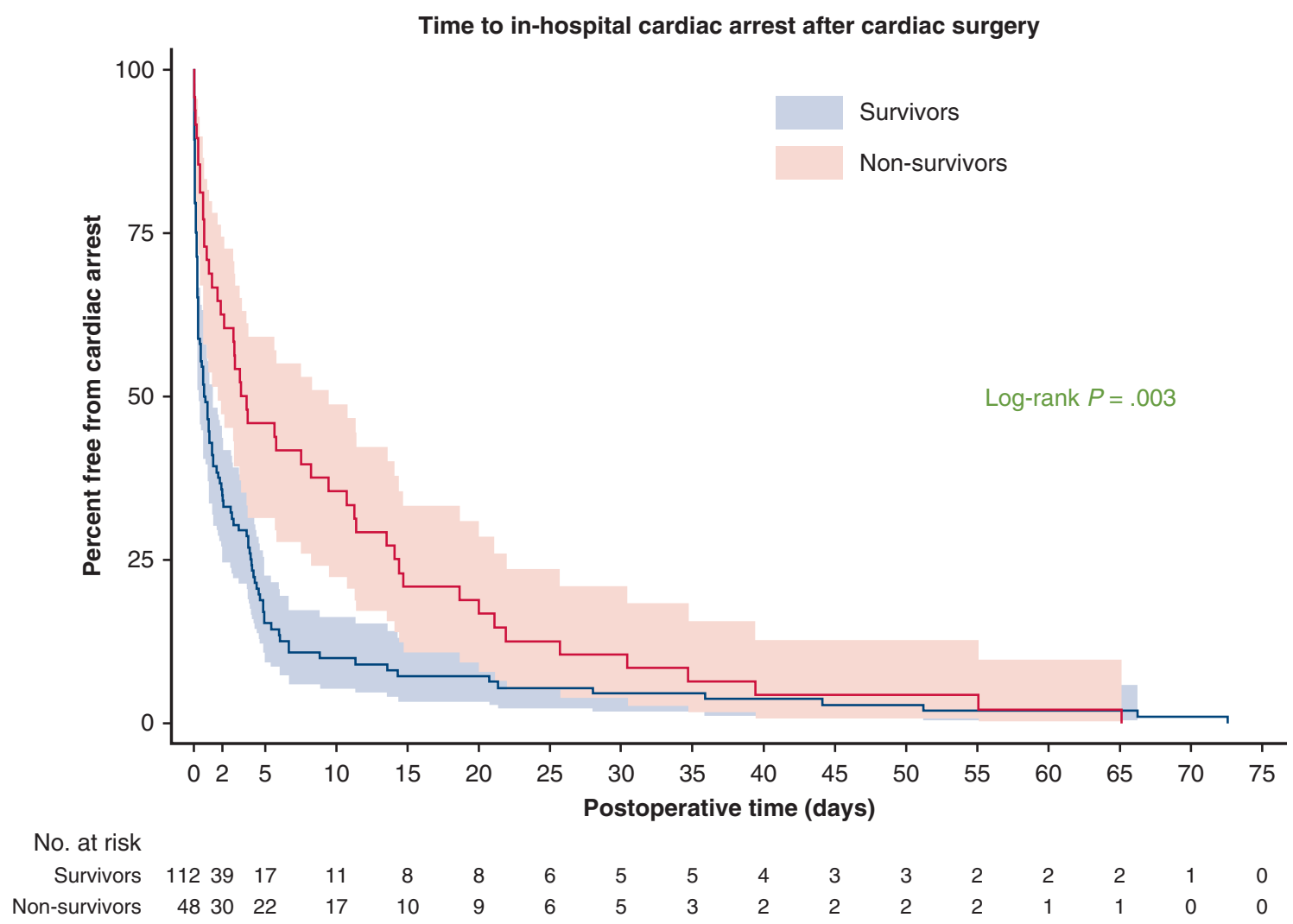

FIGURE 1. Kaplan-Meier survival curve for time to in-hospital cardiac arrest in children following cardiac surgery by suvivors versus nonsurvivors. Nonsurvivors were more likely to have experienced late arrests. The median (interquartile range [IQR]) time to arrest was 18 hours (IQR, 4-95 hours) for survivors and for nonsurvivors it was 84 hours (IQR, 17-342 hours).

resuscitation duration (median, 10 minutes [IQR, 3-25 minutes] vs 20 minutes [IQR, 10-40 minutes]; $P<.01$ ) and lower VIS (median, 5 minutes [IQR, 2-8 minutes] vs 9 minutes [IQR, 5 -18 minutes]; $P<.01$ ) (Table E1). Kaplan-Meier analysis (Figure 1) demonstrated that nonsurvivors are more likely to have had a late arrest than survivors ( $\log \operatorname{rank} P=.003)$. In multivariable analysis (reference, $<12$ hours), odds for hospital survival decreased by $65 \%$ (odds ratio, $0.35 ; 95 \%$ confidence interval, $0.10-1.2)$ in children who experienced arrest between 12 and 48 hours postsurgery and decreased by $87 \%$ (odds ratio, $0.13 ; 95 \%$ confidence interval, $0.04-0.39$ ) in children experiencing arrest $>48$ hours following surgery (Table E2).

\section{DISCUSSION}

We have demonstrated that outcomes vary with time-toarrest after cardiac surgery; therefore, overall survival rates are only partially informative. In our analysis, late arrests had an $87 \%$ reduced odds for survival compared with early arrests. The reasons are likely multifactorial: arrests occurring during the early postoperative period are commonly related to low cardiac output syndrome ${ }^{5}$ for which clear management protocols exist, children experiencing early arrests are likely to be in the closely monitored intensive care unit setting with advanced life support measures already in place ${ }^{6}$ so resuscitation can proceed quickly, and prolonged resuscitation and failure to rescue are more likely in late arrests compared with early arrests due to differing etiologies (Table 1).

Current management protocols focus on preventing major adverse events within the first 24 to 48 postoperative hours. However, our report shows more than $40 \%$ of arrests occur after 48 hours and are associated with very poor outcomes. Targeted approaches and specific protocols should be developed to identify and prevent these late arrests; in this regard, multicenter collaborative data assessing timing and etiologies of arrest may yield useful information and identify predictable patterns. The observational nature of the study and lack of long-term neurology follow-up are important limitations of our study.

\section{CONCLUSIONS}

Causes and outcomes for children who experience arrest after cardiac surgery varies with the time to arrest. This information could serve as an important metric both for quality improvement and prognostication. 


\section{References}

1. Gupta P, Wilcox A, Noel TR, Gossett JM, Rockett SR, Eble BK, et al. Characterizing cardiac arrest in children undergoing cardiac surgery: a single-center study. $J$ Thorac Cardiovasc Surg. 2017; 153:450-8.

2. Alten JAMD, Klugman DMD, Raymond TTMD, Cooper DSMD, Donohue JEM, Zhang WMS, et al. Epidemiology and outcomes of cardiac arrest in pediatric cardiac ICUs. Pediatr Crit Care Med. 2017;18:935-43.

3. Zaritsky A, Nadkarni V, Hazinski MF, Foltin G, Quan L, Wright J, et al. Recommended guidelines for uniform reporting of pediatric advanced life support: the pediatric Utstein style. Ann Emerg Med. 1995;26:487-503.
4. Gaies MG, Jeffries HE, Niebler RA, Pasquali SK, Donohue JE, Yu S, et al Vasoactive-inotropic score is associated with outcome after infant cardiac surgery: an analysis from the Pediatric Cardiac Critical Care Consortium and Virtual PICU System Registries. Pediatr Crit Care Med. 2014;15: 529-37.

5. McGuinness J, Bouchier-Hayes D, Redmond JM. Understanding the inflammatory response to cardiac surgery. Surgeon. 2008;6:162-71.

6. Ley SJ. How should we manage arrest following cardiac surgery? Semin Cardiothorac Vasc Anesth. 2015;19:87-94. 
TABLE E1. Comparison of patient and arrest characteristics between survivors and nonsurvivors at hospital discharge in children who experienced in-hospital cardiac arrest (IHCA) following cardiac surgery

\begin{tabular}{|c|c|c|c|}
\hline Characteristic & Survivors $(n=111)$ & Nonsurvivors $(n=49)$ & $P$ value \\
\hline Age (d) & $28(6-119)$ & $7(3-28)$ & $<.01$ \\
\hline Weight $(\mathrm{kg})$ & $4(3-5)$ & $3(2-4)$ & $<.01$ \\
\hline Male sex & $51(45.9)$ & $23(46.9)$ & .9 \\
\hline Prematurity* & $19(17.1)$ & $16(32.7)$ & .03 \\
\hline Chromosome/genetic syndrome & $29(26.1)$ & $10(20.4)$ & .4 \\
\hline $\begin{array}{l}\text { Time to IHCA (h) } \\
\quad<12 \\
12-48 \\
>48\end{array}$ & $\begin{array}{l}50(45.0) \\
22(19.8) \\
39(35.1)\end{array}$ & $\begin{array}{r}10(20.4) \\
9(18.4) \\
30(61.2)\end{array}$ & $<.01$ \\
\hline $\begin{array}{l}\text { RACHS-1 category } \\
1-2 \\
3-4 \\
5-6\end{array}$ & $\begin{array}{l}16(14.4) \\
75(67.6) \\
20(18.0)\end{array}$ & $\begin{array}{r}6(12.2) \\
30(61.2) \\
13(26.5)\end{array}$ & .5 \\
\hline CPB time (min) & $146(75-195)$ & $193(121-233)$ & .01 \\
\hline Aortic crossclamp time (min) & $70(24-118)$ & $63(34-112)$ & .8 \\
\hline $\begin{array}{l}\text { Location of arrest } \\
\text { PICU } \\
\text { Ward }\end{array}$ & $\begin{array}{c}101(91.0) \\
10(9.0)\end{array}$ & $\begin{array}{r}43(87.8) \\
6(12.2)\end{array}$ & .5 \\
\hline Weekend arrests $\dagger$ & $21(18.9)$ & $11(22.4)$ & 6 \\
\hline Night time arrests $\ddagger$ & $31(27.9)$ & $14(28.6)$ & .9 \\
\hline Requirement for ECPR & $44(39.6)$ & $26(53.1)$ & .1 \\
\hline CPR duration $(\min ) \S$ & $10(3-25)$ & $20(10-40)$ & $<.01$ \\
\hline Admission VIS $\S, \|$ & $5(2-8)$ & $9(5-18)$ & $<.01$ \\
\hline Admission lactate $(\mathrm{mmol} / \mathrm{L}) \S$ & $2(2-4)$ & $3(2-6)$ & $<.01$ \\
\hline ICU LOS (d) & $13(8-25)$ & $15(8-30)$ & .7 \\
\hline Hospital LOS & $37(20-76)$ & $21(13-51)$ & $<.01$ \\
\hline
\end{tabular}

Values for continuous variables (based on $\chi^{2}$ test) are presented as median (interquartile range) and categorical variables (based on Mann-Whitney rank sum test) are shown as $\mathrm{n}$ (\%). RACHS-1, risk adjusted congenital heart surgery category; $C P B$, bypass time; PICU, pediatric intensive care unit; ECPR, extracorporeal cardiopulmonary resuscitation; $C P R$, cardiopulmonary resuscitation; $V I S$, vasoactive inotropic score; $I C U$, intensive care unit; $L O S$, length of stay. *Born at $<37$ weeks' gestation. †Weekend defined as 11 pm Friday night to $7 \mathrm{am}$ Monday morning. †NNight time arrest defined as between $11 \mathrm{pm}$ and $7 \mathrm{am}$. §Missing data: CPR duration for 158 patients, admission VIS for 151 patients, an admission lactate for 148 patients. $\|$ Dopamine dose $(\mu \mathrm{g} / \mathrm{kg} / \mathrm{min})+$ dobutamine dose $(\mu \mathrm{g} / \mathrm{kg} / \mathrm{min})+100 \times$ adrenalin dose $(\mu \mathrm{g} / \mathrm{kg} / \mathrm{min})+10 \times$ milrinone dose $(\mu \mathrm{g} / \mathrm{kg} /$ $\min )+10,000 \times$ vasopressin dose $(\mathrm{U} / \mathrm{kg} / \mathrm{min})+100 \times$ noradrenalin dose $(\mu \mathrm{g} / \mathrm{kg} / \mathrm{min})$. 
TABLE E2. Logistic regression model for survival to hospital discharge in children who suffered in hospital cardiac arrest following cardiac surgery

\begin{tabular}{|c|c|c|c|c|}
\hline \multirow[b]{2}{*}{ Variable } & \multicolumn{2}{|l|}{ Unadjusted } & \multicolumn{2}{|l|}{ Adjusted } \\
\hline & Odds ratio $(95 \%$ confidence interval $)$ & $P$ value & Odds ratio $(95 \%$ confidence interval) & $P$ value \\
\hline \multicolumn{5}{|l|}{ Time to arrest (h) } \\
\hline$\leq 12$ & 1.0 & & 1.0 & \\
\hline $12-48$ & $0.49(0.17-1.4)$ & .2 & $0.35(0.10-1.2)$ & .09 \\
\hline$>48$ & $0.26(0.11-0.60)$ & $<.01$ & $0.13(0.04-0.39)$ & $<.01$ \\
\hline \multicolumn{5}{|l|}{ Age (d) } \\
\hline$\leq 28$ & 1.0 & & 1.0 & \\
\hline$>28$ & $2.9(1.4-6.2)$ & $<.01$ & $6.8(2.3-20)$ & $<.01$ \\
\hline \multicolumn{5}{|l|}{ Admission VIS* } \\
\hline$<10$ & 1.0 & & 1.0 & \\
\hline$>10$ & $0.33(0.15-0.70)$ & $<.01$ & $0.29(0.11-0.74)$ & .01 \\
\hline \multicolumn{5}{|l|}{ RACHS- 1 category } \\
\hline $1-2$ & 1.0 & & 1.0 & \\
\hline $3-4$ & $0.94(0.33-2.6)$ & .9 & $3.5(0.91-13.4)$ & .07 \\
\hline $5-6$ & $0.58(0.18-1.9)$ & .4 & $9.3(1.6-56)$ & .01 \\
\hline \multicolumn{5}{|c|}{ CPB duration (min) } \\
\hline$<90$ & 1.0 & & 1.0 & \\
\hline $90-180$ & $0.80(0.30-2.1)$ & .7 & $0.59(0.19-1.8)$ & .4 \\
\hline$>180$ & $0.31(0.12-0.79)$ & .01 & $0.25(0.07-0.86)$ & .03 \\
\hline \multicolumn{5}{|l|}{ Location of arrest } \\
\hline PICU & 1.0 & & NS & \\
\hline Ward & $0.84(0.49-1.4)$ & .5 & & \\
\hline Prematurity (wk) & & & NS & \\
\hline$\geq 37$ & 1.0 & & & \\
\hline$<37$ & $0.41(0.19-0.89)$ & .02 & & \\
\hline
\end{tabular}

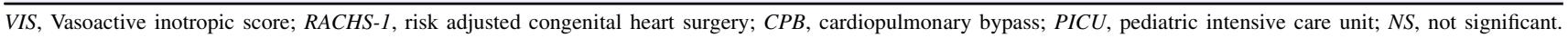
$* \mathrm{n}=151$ (rather than 160) due to missing admission VIS data. 